\title{
Para ti, información práctica
}

\section{For you, practical information}

En esta sección se presentan las guías prácticas y documentos de consenso más actuales publicados en el mundo para ayudar a los dietistas-nutricionistas y, en general, a todos los profesionales de la salud en la toma de decisiones en materia de nutrición humana y dietética. El Comité Editorial ha realizado una búsqueda de todas las guías y los documentos de consenso publicados entre enero y marzo de 2012 (incluidos algunos documentos importantes de 2011 recientemente hallados) en el apartado de publicaciones sobre nutrición de la Organización Mundial de la Salud (www.who.int), el Bulletin of the World Health Organization (http://www. who.int/ bulletin/en/), los apartados de Salud Pública y de Nutrición y Actividad Física de la Unión Europea (http://ec.europa. eu/), el apartado de legislación de la Unión Europea (http://europa.eu/legislation_summaries/index_en.htm), el apartado de publicaciones de la Autoridad Europea de Seguridad Alimentaria (EFSA) (www.efsa.europa.eu), el apartado de publicaciones de la Agencia Española de Seguridad Alimentaria y Nutrición (AESAN) (www.aesan.msc.es), la National Guideline Clearinghouse (NGC) (www.guideline.gov) y MEDLINE-PubMed (www.pubmed.gov).

Nota importante: Recomendamos a todos los lectores que consulten esta sección a través de la página web www.elsevier.es/dietetica para que el acceso a los textos completos sea más sencillo.

\section{Organización Mundial de la Salud (OMS)}

Suplementación intermitente de hierro en niños preescolares y en edad escolar (guía \#1) y suplementación intermitente de hierro y ácido fólico en mujeres que menstrúan (guía \#2)

Estas dos guías ofrecen recomendaciones basadas en la evidencia sobre el uso intermitente de suplementos de hierro en ambos subgrupos de población y de ácido fólico en mujeres que menstrúan como medida de salud pública para mejorar la concentración de hierro y disminuir el riesgo de anemia. La guía \#2 sustituye una declaración anterior, pu- blicada en 2009, sobre la suplementación semanal de hierro y ácido fólico para mujeres en edad reproductiva.

Texto completo guía \#1: http://whqlibdoc.who.int/ publications/2011/9789241502023_eng.pdf

Texto completo guía \#2:http://whqlibdoc.who.int/ publications/2011/9789241502009_eng.pdf

\section{Utilización en el hogar de múltiples micronutrientes en polvo para fortificar alimentos consumidos por los lactantes y niños de 6-23 meses de edad (guía \#1) y por mujeres embarazadas (guía \#2)}

Estas dos guías ofrecen recomendaciones basadas en la evidencia sobre el uso de múltiples micronutrientes en polvo para fortificar en el hogar alimentos consumidos por ambos subgrupos de población.

Texto completo: http: / /whqlibdoc.who.int/publications/ 2011/9789241502047_eng.pdf

\section{Evaluación de residuos de fármacos veterinarios en los alimentos}

Texto completo: http://whqlibdoc.who.int/publications/ 2012/9789241209694_eng.pdf

Consulta técnica de la OMS sobre vitamina A en la salud de los recién nacidos: estudios mecanísticos

Texto completo: http://whqlibdoc.who.int/publications/ 2012/9789241503167_eng.pdf

La evaluación de las intervenciones en los sistemas sanitarios: aspectos clave al considerar el valor de la aleatorización

Se necesita realizar estudios para facilitar la identificación de intervenciones que mejoren la capacidad o el funciona- 
miento de los sistemas sanitarios y, por lo tanto, contribuir a lograr las metas de salud general. Cuando se realizan correctamente, los estudios controlados aleatorizados (ECA) proporcionan la más sólida evidencia para identificar que las intervenciones brindadas directamente a las personas son seguras y eficaces. No obstante, surgen desafíos adicionales cuando la investigación se centra en intervenciones basadas en múltiples unidades de organización encontradas dentro de los sistemas sanitarios. Por lo tanto, no se puede suponer que la aleatorización pueda reducir o eliminar el sesgo y la confusión en el mismo grado en todos los casos. Si bien otros autores tienen argumentos expuestos a favor de diseños alternativos, en este documento el objetivo es ayudar a entender por qué puede verse amenazado el valor potencial de los ECA. Específicamente, propone cinco puntos que tener en cuenta al explorar los desafíos del diseño o la evaluación de los ECA en las intervenciones en sistemas sanitarios: a) el número de las unidades disponibles para aleatorización; b) la complejidad de la unidad organizativa en estudio; c) la complejidad de la intervención; d) la complejidad de la relación de causa y efecto, y e) la contaminación. Además, los autores señalan que la heterogeneidad de los resultados puede ser informativa y que se debe explorar y no ignorar las razones de dicho fenómeno.

Bull World Health Organ. 2011;89:907-12.

Texto completo: http://www.who.int/bulletin/volumes/ 89/12/11-089524.pdf

Desempeño diagnóstico de la emaciación grave visible (edema nutricional) para identificar la desnutrición aguda grave en niños ingresados en hospitales de Kenia

Objetivo: Determinar el valor diagnóstico de la emaciación grave visible en la identificación de la desnutrición aguda grave en dos hospitales públicos en Kenia. Conclusiones: Se falló en la detección de la emaciación grave visible en aproximadamente la mitad de los niños ingresados en el hospital con un diagnóstico antropométrico de desnutrición aguda. La evaluación sistemática por MUAC es rápida, simple y económica y debería formar parte de la evaluación estándar de todos los ingresos pediátricos en el centro de estudio.

Bull World Health Organ. 2011;89:900-6.

Texto completo: http: / /www.who.int/bulletin/volumes/ 89/12/11-091280.pdf

\section{Colaboración para optimizar las ingestas dietéticas de sal y yodo: un problema crítico de salud pública pero en ocasiones pasado por alto}

En este breve artículo que aparece en la sección "Perpective" del Boletín de la OMS de enero de 2012, se desarrolla una interesante reflexión acerca de cómo las campañas de reducción del consumo de sal y las campañas de suplementación de yodo a través de las sales pueden entrar en conflicto. Los autores insisten en que ambas campañas pueden realizarse simultáneamente, pero que es necesaria la colaboración entre los agentes gubernamentales que las lideran. Bull World Health Organ. 2012;90:73-4.
Texto completo: http://www.who.int/bulletin/volumes/ 90/1/11-092080.pdf

\section{Unión Europea (UE)}

Informe de la Comisión al Parlamento Europeo y al Consejo relativo a los alimentos e ingredientes tratados con radiaciones ionizantes en el año 2010

Texto completo: http://eur-lex.europa.eu/LexUriServ/ LexUriServ.do?uri=COM:2012:0017:FIN:ES:PDF

\section{Cambios en la legislación que regula los "productos o alimentos dietéticos"}

El objetivo de la propuesta de la UE es revisar la legislación sobre los productos alimenticios destinados a una alimentación especial incluidos en la Directiva 2009/39/CE, la denominada "Directiva marco sobre alimentos dietéticos".

La UE ha advertido de que "[...] algunos explotadores del sector notifican alimentos normales en virtud de la legislación sobre alimentos dietéticos a fin de poder utilizar la indicación de adecuación dietética (obligatoria conforme a lo dispuesto en la legislación en la materia) en vez de la declaración nutricional correspondiente, y así eludir los requisitos previstos en el Reglamento sobre declaraciones nutricionales y propiedades saludables (autorización previa de la UE con evaluación científica). Esta situación produce una distorsión del mercado y da lugar a condiciones de competencia desiguales para los operadores [...]".

En consecuencia, tras un estudio minucioso de las implicaciones socioeconómicas de cuatro opciones diferentes, se ha propuesto:

- Derogar la normativa marco (Directiva 2009/39/CE): esto evitará la diferenciación entre "producto o alimento dietético" y "alimento normal" y hará que todos los alimentos tengan la misma consideración en la legislación general y, en consecuencia, tengan los mismos requisitos a la hora de demostrar declaraciones nutricionales y de propiedades saludables (Reglamento Europeo 1924/2006).

- Mantener o incorporar algunas de las normas específicas adoptadas en virtud de este marco y que han aportado un valor añadido a escala europea en cuanto a armonización, claridad y protección al consumidor.

Textos completos: Propuesta de Reglamento del Parlamento Europeo y del Consejo relativo a los alimentos destinados a los lactantes y niños de corta edad y los alimentos para usos médicos especiales: http://eur-lex.europa.eu/ LexUriServ/LexUriServ.do?uri=COM:2011:0353:FIN:ES:PDF

Documento de trabajo de los Servicios de la Comisión, resumen de la evaluación de impacto que acompaña al documento "Propuesta de Reglamento del Parlamento Europeo y del Consejo sobre alimentos destinados a lactantes y niños de corta edad y alimentos destinados a usos médicos especiales": http://eur-lex.europa.eu/LexUriServ/LexUriServ.do?uri=SEC:2011:0763:FIN:ES:PDF 
Sesión plenaria de la Plataforma Europea para la Acción sobre la Dieta, la Actividad Física y la Salud, Bruselas, 9 de febrero de 2012

Ya están disponibles las presentaciones y los documentos derivados de la reunión anual de la Plataforma Europea para la Acción sobre la Dieta, la Actividad Física y la Salud: "Impuestos sobre los alimentos: estado actual", "Iniciativas Comunitarias dirigidas a la Obesidad Infantil", "Iniciativa de Vigilancia de la Obesidad Infantil de la OMS", "NU-AGE: Nuevas iniciativas dietéticas para abordar las necesidades específicas de la población de edad avanzada para un envejecimiento saludable", "Directiva de servicios de medios audiovisuales: informe sobre su aplicación", "Resumen de los compromisos en el campo de la reformulación", "Los ácidos grasos trans: iniciativa de Alemania", "Guía FoodDrinkEurope en el tamaño de las porciones", "Innovación de productos y renovación de Nestlé" y "Reformulación de McCain Food Europe".

Documentos disponibles en: http: / /ec.europa.eu/health/ nutrition_physical_activity/events/ev_20120209_en.htm

\section{European Food Safety Authority (EFSA)}

Opinión científica sobre la declaración de propiedades saludables de la fibra de remolacha, el aumento del volume fecal y la disminución del tiempo de tránsito intestinal con arreglo al Artículo 13(5) del Reglamento (EC) No 1924/2006

Alimento/ingrediente/sustancia/dieta sujeta a la declaración de salud: fibra de remolacha. Declaración de salud: "aumento del volumen fecal" y "disminución del tiempo de tránsito intestinal”. Población diana: población general. Conclusión: el Panel concluye que se ha establecido una relación causa-efecto entre la ingesta de fibra de la remolacha y aumento del volumen fecal, pero no con la disminución del tiempo de tránsito intestinal. Para poder hacer dicha declaración de salud, el alimento debe ser "alto en fibra" según los criterios definidos en el Anexo del Reglamento $1924 / 2006$.

Texto completo: http://www.efsa.europa.eu/en/efsajournal/doc/2468.pdf http://www.efsa.europa.eu/en/efsajournal/doc/2467. pdf

Opinión científica sobre la declaración de propiedades saludables de la glucosamina y el mantenimiento de las articulaciones con arreglo al Artículo 13(5) del Reglamento (EC) No 1924/2006

Alimento/ingrediente/sustancia/dieta sujeta a la declaración de salud: glucosamina. Declaración de salud: "contribuye a la protección del cartilage de las articulaciones expuestas a un movimiento o carga excesiva, y ayuda a mejorar el rango de movimiento de las mismas". Población diana: individuos saludables expuestos a una carga excesiva de las articulaciones. Conclusión: el Panel concluye que no se ha establecido relación causa-efecto entre la ingesta de glucosamina y "el mantenimiento de las articulaciones".
Texto completo: http://www.efsa.europa.eu/en/efsajournal/doc/2476.pdf

Opinión científica sobre la declaración de propiedades saludables del extracto de la proteína de soja y la reducción de las concentraciones sanguíneas de LDL-colesterol con arreglo al Artículo 13(5) del Reglamento (EC) No 1924/2006

Alimento/ingrediente/sustancia/dieta sujeta a la declaración de salud: extracto de proteína de soja. Declaración de salud: "reducción de las concentraciones sanguíneas de LDL-colesterol". Población diana: sujetos sanos con concentraciones sanguíneas de colesterol normales o ligeramente elevadas. Conclusión: el Panel concluye que no se ha establecido relación causa-efecto entre la ingesta de extracto de proteína de soja y la reducción de las concentraciones sanguíneas de colesterol de las lipoproteínas de baja densidad.

Texto completo: http://www.efsa.europa.eu/en/ efsajournal/doc/2555.pdf

Opinión científica sobre la declaración de propiedades saludables del aceite diacilglicerol (DAG) y la reducción del peso corporal con arreglo al Artículo 13(5) del Reglamento (EC) No $1924 / 2006$

Alimento/ingrediente/sustancia/dieta sujeta a la declaración de salud: aceite diacilglicerol (DAG) de origen vegetal con al menos el $80 \%$ diacilglicerol, que sustituye a otros aceites vegetales de parecida composición en ácidos grasos que contienen, en su mayoría (> 90\%), triacilglicerol (TAG). Declaración de salud: "ayuda en el manejo del peso corporal mediante la disminución de peso". Población diana: población general. Conclusión: el Panel concluye que no se ha establecido relación causa-efecto entre la ingesta de aceite diacilglicerol (DAG) y la "reducción del peso corporal".

Texto completo:http://www.efsa.europa.eu/en/ efsajournal/doc/2469.pdf

Opinión científica sobre la declaración de propiedades saludables de "snacks hipocalóricos (productos KOT)" y la "contribución a la reducción del tamaño de los adipocitos a nivel abdominal en el contexto de una dieta baja en calorías" con arreglo al Artículo 13(5) del Reglamento (EC) No $1924 / 2006$

Alimento/ingrediente/sustancia/dieta sujeta a la declaración de salud: snacks hipocalóricos. Declaración de salud: "contribución a la reducción del tamaño de los adipocitos a nivel abdominal en el contexto de una dieta baja en calorías". Población diana: individuos con sobrepeso que quieren reducer su grasa abdominal. Conclusión: el Panel considera que las evidencias aportadas no establecen que la reducción del tamaño de los adipocitos subcutáneos abdominales sea, per se, un efecto beneficioso para la salud.

Texto completo: http://www.efsa.europa.eu/en/ efsajournal/doc/2381.pdf 
Opinión científica de la EFSA sobre los Valores Dietéticos de Referencia (VDR) para proteínas

Texto completo: http://www.efsa.europa.eu/en/ supporting/doc/225e.pdf

\section{Agencia Española de Seguridad Alimentaria y Nutrición (AESAN)}

Productos alimenticios apropiados para personas con intolerancia al gluten

A partir del 1 de enero de 2012 resultarán obligatorias en toda la UE las disposiciones del Reglamento (CE) N. ${ }^{\circ} 41 / 2009$ sobre composición y etiquetado de productos alimenticios para personas con intolerancia al gluten.

Texto completo Reglamento 41/2009: http: / / eur-lex.europa.eu/LexUriServ/LexUriServ.do?uri=OJ:L:2009:016:0003 :0005:ES:PDF

Publicación del Reglamento 1169/2011

sobre la información alimentaria facilitada

al consumidor

El texto modifica los Reglamentos (CE) N. ${ }^{\circ} 1924 / 2006$ y (CE) N. ${ }^{\circ} 1925 / 2006$ del Parlamento Europeo y del Consejo, y deroga la Directiva $87 / 250 /$ CEE de la Comisión, la Directiva 90/496/CEE del Consejo, la Directiva 1999/10/CE de la Comisión, la Directiva 2000/13/CE del Parlamento Europeo y del Consejo, las Directivas 2002/67/CE y 2008/5/CE de la Comisión, y el Reglamento (CE) N. ${ }^{\circ}$ 608/2004 de la Comisión.

Texto completo del Reglamento 1169/2011:

http: / / eur-lex.europa.eu/LexUriServ/LexUriServ.do?uri=0 J:L:2011:304:0018:0063:ES:PDF

Informe del Comité Científico de la Agencia Española de Seguridad Alimentaria y Nutrición (AESAN) en relación con la evaluación del riesgo de la exposición de lactantes y niños de corta edad a nitratos por consumo de acelgas en España (Revista del Comité Científico de la AESAN N. ${ }^{\circ} 14$ ).

Texto completo:http://www.aesan.msc.es/AESAN/docs/ docs/publicaciones_estudios/revistas/comite_cientifico_14.pdf

\section{National Guideline Clearinghouse (NGC)}

\section{Estilos de vida saludables}

Healthy lifestyles. Bloomington: Institute for Clinical Systems Improvement (ICSI); 2011.

Resumen de guideline.gov: http://www.guideline.gov/ content. aspx?id=34021

Texto completo: http://www.icsi.org/chronic_disease_ risk_factors_primary_prevention_of_guideline__23506/ chronic_disease_risk_factors_primary_prevention_of guideline_23508.html

\section{Enfermedad coronaria estable}

Stable coronary artery disease. Bloomington: Institute for Clinical Systems Improvement (ICSI); 2011.

Resumen de guideline.gov: http://www.guideline.gov/ content. aspx?id=32826

Texto completo: http://www.icsi.org/coronary_artery_ disease/coronary_artery_disease_stable_.html

Guía Basada en la Efectividad para la prevención de las enfermedades cardiovasculares en mujeres, actualización 2011: una guía de la American Heart Association (AHA)

http://circ.ahajournals.org/content/123/11/1243.full.pdf

\section{MEDLINE-PubMed}

Revisión sistemática sobre las intervenciones de apoyo a la continuación de la lactancia materna: un análisis de metarregresión de los efectos, la intensidad y el tiempo

Resumen PubMed: http://www.ncbi.nlm.nih.gov/pubmed/22277543

Texto completo: http://www.bmj.com/highwire/filestream/562027/field_highwire_article_pdf/0.pdf

Guías de la American Cancer Society de nutrición y actividad física para la prevención del cáncer: reduciendo el riesgo de cáncer con elección saludable de alimentos y actividad física

Resumen PubMed: http://www.ncbi.nlm.nih.gov/pubmed/ 22237782

Texto completo: http://onlinelibrary.wiley.com/doi/ 10.3322/caac. 20140/pdf

Ingesta de magnesio dietético y riesgo de accidente cerebrovascular (ACV): metaanálisis de estudios prospectivos

Conclusión: la ingesta de $100 \mathrm{mg} /$ día de magnesio dietético se asocia inversamente con el riesgo de ACV (reducción del $8 \%$ ).

Resumen PubMed: http://www.ncbi.nlm.nih.gov/pub$\mathrm{med} / 22205313$

Tratamiento dietético de la alergia a la proteína de leche de vaca en niños: un comentario del Committee on Nutrition of the French Society of Paediatrics

Resumen PubMed: http://www.ncbi.nlm.nih.gov/pubmed/ 22115523

Vitamina A y riesgo de cáncer cervical: metaanálisis

Resumen PubMed: http://www.ncbi.nlm.nih.gov/pubmed/ 22005522 